\title{
Loss of mass and performance in skeletal muscle tissue: a continuum model
}

\author{
Giulia Giantesio $^{1}$, Alfredo Marzocchi ${ }^{1}$, Alessandro Musesti ${ }^{1 *}$ \\ Dipartimento di Matematica e Fisica, \\ Università Cattolica del Sacro Cuore, Brescia \\ Via dei Musei 41, 21521 Brescia, Italy \\ *Email address for correspondence: alessandro.musesti@unicatt.it \\ Communicated by Giorgio Fotia \\ Received on 12 16, 2016. Accepted on 12 18, 2017.
}

\begin{abstract}
We present a continuum hyperelastic model which describes the mechanical response of a skeletal muscle tissue when its strength and mass are reduced by aging. Such a reduction is typical of a geriatric syndrome called sarcopenia. The passive behavior of the material is described by a hyperelastic, polyconvex, transversely isotropic strain energy function, and the activation of the muscle is modeled by the so called active strain approach. The loss of ability of activating of an elder muscle is then obtained by lowering of some percentage the active part of the stress, while the loss of mass is modeled through a multiplicative decomposition of the deformation gradient. The obtained stress-strain relations are graphically represented and discussed in order to study some of the effects of sarcopenia.

Keywords: sarcopenia, active strain, hyperelasticity, damage

AMS subject classification: 74B20, 74L15
\end{abstract}

\section{Introduction}

Skeletal muscle tissue, whose principal role is the production of force and hence movement, is a highly-ordered hierarchical structure. Muscular fibers, the cells of the tissue, are organized in fascicles, where every fiber is multiply connected to nerve axons. They contain a concatenation of millions of sarcomeres, which are the fundamental unit of the muscle and the actuators of activation, the mechanism by which a muscle produces force.

The aim of this paper is to propose a mathematical model of skeletal muscle tissue which can exhibit a reduced performance and mass, typical of a geriatric syndrome named sarcopenia [1], defined as the loss of skeletal muscle mass and strength that occurs with advancing age. Although nowa- 
days the syndrome affects more than 50 millions people, so far there are no compelling tests for its diagnosis and many efforts are made by the medical community to better understand it. Therefore it is desirable to build a mathematical model of muscle tissue affected by sarcopenia. As far as we know, the first elastic model of sarcopenic skeletal muscle has been proposed in [2]; however, that paper focuses only on the loss of performance. Here we generalize that model, including also the loss of mass.

In view of the highly organized structure of the fibers in skeletal muscles, from a macroscopic viewpoint it is reasonable to model the tissue as a continuum material which is transversely isotropic. Moreover, since the tissue is composed mainly of water, we will assume incompressibility. Finally, focusing our attention only on steady properties, we neglect the viscous effects and set in the framework of hyperelasticity. In the model that we propose, there are two main constitutive prescriptions: one for the hyperelastic energy when the tissue is not activated (passive energy) and one for the activation. Moreover, in order to describe sarcopenia, the loss of performance and the loss of mass are obtained by two different methods.

As far as the passive part is concerned, we assume an exponential stress response of the material, which is customary in biological tissues. The hyperelastic strain energy density takes into account also the isotropic and anisotropic components of the tissue.

Coming to activation, a recent and very promising way to describe it is the so called active strain approach [3], where the extra energy produced by the activation mechanism is encoded in a multiplicative decomposition of the deformation gradient in an elastic and an active part. Unlike the classical active stress approach, in which the active part of the stress is modeled in a pure phenomenological way and a new term has to be added to the passive energy, the active strain method does not change the form of the elastic energy, keeping in particular all its mathematical properties. Furthermore, in the case of muscles the active strain approach is more adherent to the physiology of the tissue, being a mathematical representation of the contraction of the sarcomeres at the molecular level. The active strain approach has firstly been applied to the skeletal muscle tissue in [2], where the active strain is described by a parameter which depends on the deformation. One of the novelties of the present paper is to give an active model which is completely hyperelastic, in the sense that the active stress can be computed as the derivative with respect to the deformation gradient of a suitable strain energy function. See also [4] for a detailed discussion on this issue.

Another feature of our model is the description of the two main effects of sarcopenia: the loss of activation and the loss of muscle mass. The former 
is represented by a damage parameter $d$ which reduces the active part of the stress by a given percentage. The latter is encoded in the model by a further multiplicative decomposition of the deformation gradient, where a parameter $g$ measures the fraction of muscle fibers which are not active anymore.

We present some numerical results, showing that the experimental data of [5] on the passive and active stress-strain curves, obtained in vivo from a tetanized tibialis anterior of a rat, can be well reproduced by our model in the healthy case. The lack of any experiment on the elastic properties of a sarcopenic muscle tissue does not allow any fitting of the two damage parameters $d$ and $g$; however, different scenarios are studied when $d$ and $g$ increase.

The model here proposed can be numerically implemented using finite element methods. In the future, it will be very interesting to perform numerical simulations on a realistic mesh and to find some connections between the damage percentages (parameters $d$ and $g$ ) and other physiological quantities.

\section{Constitutive model}

Skeletal muscle tissue gives our body its shape and its ability to produce movement and force. Even if the tissue is composed mainly by water $(72-75 \%$ amount), its structure is very complex and the tension developed during elongation is the combination of the presence of connective tissue and fibers. Connective tissue, which is essentially isotropic, fills the spaces among the fibers and it is responsible of the elastic recoil of the muscle to elongation, while the fibers, having a preferred alignment, break the isotropy of the tissue and drive the activation.

Let us analyze a typical stress-strain relation such as the one given in Fig. 1, which shows the data obtained by Hawkins and Bey [5] on the relation between the stretch along the fibers and the developed stress of a tetanized rat tibialis anterior. Due to the elastic behavior of the tissue, as a muscle is stretched in absence of activation, it starts to resist, very weakly for small elongations and much more strongly for large deformations (passive curve). When the tissue is stimulated, the presence of actin and myosin in the fibers allows the muscle to activate, changing considerably the shape of the stress-strain relation (total curve). This behavior depends on the number of the cross-bridges formed by the sliding movement of actin on myosin. By subtracting the passive force from the results of the stimulated test, we can isolate the contribution due to the contractile elements (active curve). 


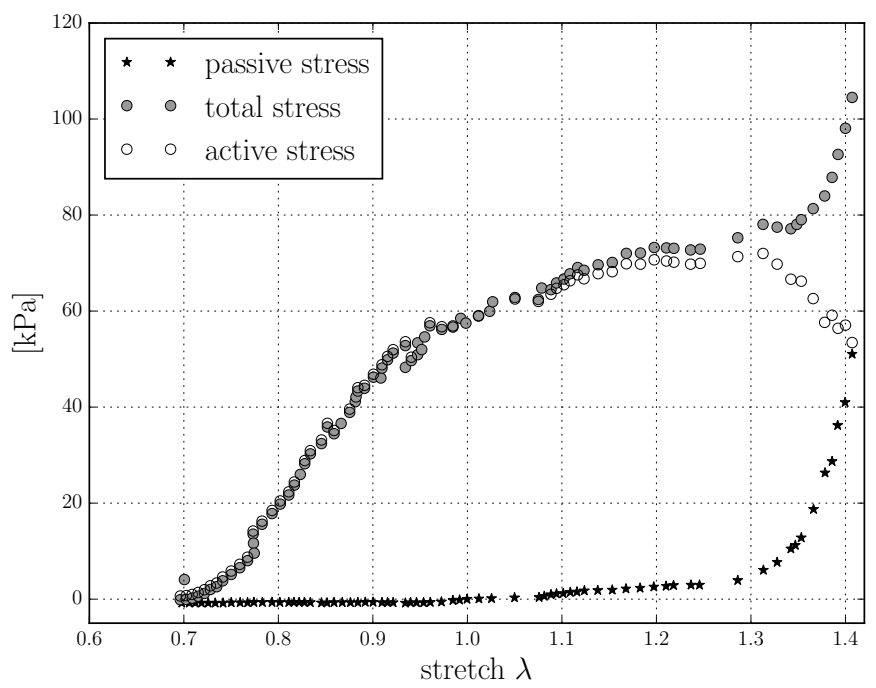

Figure 1. Stress-stretch relationship reported in [5].

We recall that the experiment of Hawkins and Bey is performed on disarticulated muscles, so that the range of the stretch is not physiological. An intact human muscle usually functions in the central portion of the stress-strain curve, although the precise shape of the curve varies across muscles [6].

Having the previous considerations in mind, it is important to include in our mathematical model the combination of the effects due to both the connective tissue and the presence of the fibers.

\subsection{Passive model}

In Continuum Mechanics the motion of a body is described by an invertible smooth map from a bounded subset $\Omega \subset \mathbb{R}^{3}$ into $\mathbb{R}^{3}$ : the function $\mathbf{x}=\chi(\mathbf{X}, t)$ associates every point $\mathbf{X}$ in the reference configuration $\Omega$ with its current placement $\mathbf{x}$. The deformation gradient

$$
\mathrm{F}=\operatorname{Grad} \chi, \quad F_{i j}=\frac{\partial x_{i}}{\partial X_{j}}, \quad i, j=1,2,3
$$

belongs to the space of linear operators with strictly positive determinant.

In the following, we set in the frame of hyperelasticity. For a hyperelastic material, the first Piola-Kirchhoff (or nominal) stress tensor P, which describes the tensional state in a continuum medium, is derived from a strain 
energy density function $W$. By frame-indifference, $W$ is a function of the right Cauchy-Green tensor $\mathrm{C}=\mathrm{F}^{T} \mathrm{~F}$, so that

$$
\mathrm{P}(\mathbf{X}, \mathrm{F}(\mathbf{X}))=2 \mathrm{~F}(\mathbf{X}) \frac{\partial W}{\partial \mathrm{C}}(\mathbf{X}, \mathbf{C}(\mathbf{X})) .
$$

Hence the behavior of the elastic body is described by the hyperelastic strain energy function

$$
\int_{\Omega} W(\mathbf{X}, \mathbf{C}(\mathbf{X})) d \mathbf{X}
$$

From now on we assume that the material is homogeneous, so that $W$ does not depend explicitly on $\mathbf{X}$.

As is customary for skeletal muscle tissue, we assume that the material is incompressible, due to the total amount of water in the tissue, and transversely isotropic, thanks to the alignment of the fibers. Following [2], we model the passive behavior of the material by the strain energy density function

$$
W(\mathrm{C})=\frac{\mu}{4}\left\{\frac{1}{\alpha}\left[e^{\alpha\left(I_{p}-1\right)}-1\right]+K_{p}-1\right\},
$$

where

$$
I_{p}=\frac{w_{0}}{3} \operatorname{tr}(\mathbf{C})+\left(1-w_{0}\right) \operatorname{tr}(\mathrm{CM}), \quad K_{p}=\frac{w_{0}}{3} \operatorname{tr}\left(\mathrm{C}^{-1}\right)+\left(1-w_{0}\right) \operatorname{tr}\left(\mathrm{C}^{-1} \mathrm{M}\right),
$$

together with the incompressibility constraint

$$
\operatorname{det} C=1 .
$$

The tensor $\mathbf{M}=\mathbf{m} \otimes \mathbf{m}$ is called structural tensor, $\mathbf{m}$ being the local orientation of the fibers, $\mu$ is an elastic coefficient, $\alpha$ and $w_{0}$ are positive dimensionless material constants. The generalized invariants $I_{p}$ and $K_{p}$ are a weighted combination of the isotropic and anisotropic components; in particular, $w_{0}$ measures the ratio of isotropic tissue constituents and $1-w_{0}$ that of muscle fibers. Moreover, the term $\operatorname{tr}(\mathrm{CM})$ represents the squared stretch in the direction of $\mathbf{m}$ and is thus associated with the elongation of the fibers, while the term $\operatorname{tr}\left(\mathrm{C}^{-1} \mathrm{M}\right)$ describes the change of the crosssectional area of a surface element which is normal to the direction $\mathbf{m}$ in the reference configuration and thus relates to the transverse behavior of the material $[7,8]$.

The Piola-Kirchhoff stress tensor is then given by

$$
\begin{aligned}
\mathrm{P}=2 \mathrm{~F} \frac{\partial W}{\partial \mathrm{C}}-p \mathrm{~F}^{-T}=\frac{\mu}{2} \mathrm{~F}\{ & e^{\alpha\left(I_{p}-1\right)}\left[\frac{w_{0}}{3} \mathrm{I}+\left(1-w_{0}\right) \mathrm{M}\right] \\
& \left.-\mathrm{C}^{-1}\left[\frac{w_{0}}{3} \mathrm{I}+\left(1-w_{0}\right) \mathrm{M}\right] \mathrm{C}^{-1}\right\}-p \mathrm{~F}^{-T}
\end{aligned}
$$


where $p$ is a Lagrange multiplier associated with the hydrostatic pressure due to incompressibility.

The strain energy density function (4) is a slight simplification of the one proposed in [9]. One of the mathematical features of (4) is that it is polyconvex and coercive $[7,10]$, hence the equilibrium problem with mixed boundary conditions is well posed. We remark that $\mathrm{C}$ is the identity tensor I in the reference configuration, so that $I_{p}=K_{p}=1$, i.e. we have the energyand stress-free state of the passive muscle tissue.

The material parameters of the model can be obtained from real data. More precisely, concerning the elastic coefficient, we use the value $\mu=0.1599 \mathrm{kPa}$ given in [9], while the other two material constants have been obtained by least squares optimization using the experimental data by Hawkins and Bey [5] about the stretch response of a tetanized tibialis anterior of a rat, see Fig. 2.

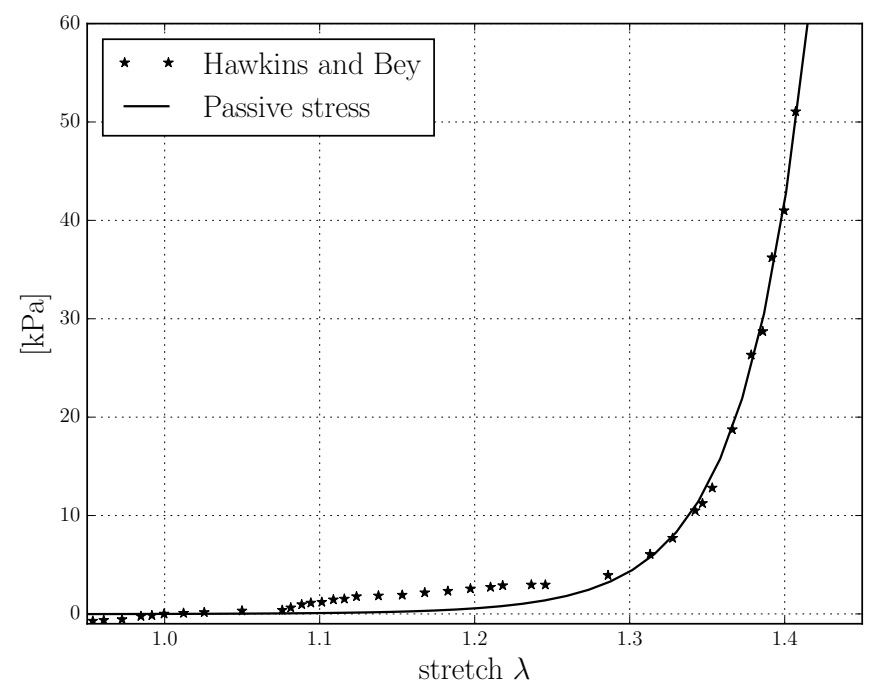

Figure 2. Passive stress along the fibers $\left(\mu=0.1599 \mathrm{kPa}, \alpha=19.35, w_{0}=0.7335\right)$.

\subsection{Active model}

One of the main features of the muscle tissue is its ability of activating through a chemical reaction between the actin and myosin filaments, which induces a contraction of the muscle fibers. In this section we introduce a parameter which describes the active state of the tissue in the hyperelastic energy. 


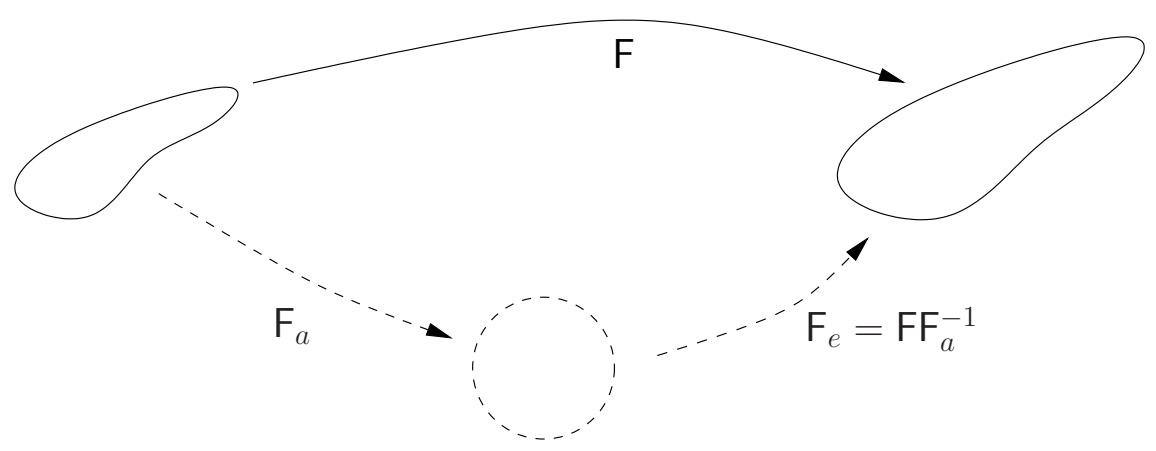

Figure 3. Kröner-Lee decomposition for the active strain approach.

Following the active strain approach [2], we rewrite the deformation gradient with the Kröner-Lee decomposition

$$
\mathrm{F}=\mathrm{F}_{e} \mathrm{~F}_{a}
$$

where $F_{e}$ is the elastic part and $F_{a}$ describes the active contribution (see Fig. 3). The active strain $F_{a}$ represents a change of the reference volume elements due to the contraction of the sarcomeres, so that it does not store any elastic energy. A reference volume element, distorted by $F_{a}$, needs a further deformation $\mathrm{F}_{e}$ to match the actual volume element, which accommodates both the external forces and the active contraction. Notice that neither $\mathrm{F}_{a}$ nor $\mathrm{F}_{e}$ need to be the gradients of some displacement, that is, it is not necessary that they fulfill the compatibility condition $\operatorname{curl} F_{a}=0$ or $\operatorname{curl} \mathrm{F}_{e}=0$.

The volume elements are modified by the internal active forces without changing the elastic energy, hence the strain energy function of the activated material has to be computed using $\mathrm{C}_{e}=\mathrm{F}_{e}^{T} \mathrm{~F}_{e}$ and taking into account $\mathrm{F}_{e}=\mathrm{FF}_{a}^{-1}$. We then obtain the total hyperelastic energy density

$$
\widehat{W}(\mathrm{C})=\left(\operatorname{det} \mathrm{F}_{a}\right) W\left(\mathrm{C}_{e}\right)=\left(\operatorname{det} \mathrm{F}_{a}\right) W\left(\mathrm{~F}_{a}^{-T} \mathrm{CF}_{a}^{-1}\right) .
$$

We remark that the active strain approach was first proposed by Taber and Perucchio [3] for cardiac tissue and it has been developed by several authors (see for example $[2,11-14]$ ). Differently from the more popular $a c$ tive stress approach, this method does not change the form of the strain energy function and it seems to be more related to the biological meaning of activation [12].

Now we have to constitutively provide the form of the active part $F_{a}$. Since the muscle contracts along the fibers, it is reasonable to describe the 
activation as

$$
\mathrm{F}_{a}=(1-a) \mathbf{m} \otimes \mathbf{m}+\frac{1}{\sqrt{1-a}}(\mathbf{I}-\mathbf{m} \otimes \mathbf{m})
$$

where $0 \leq a<1$ is a dimensionless parameter representing the relative contraction of activated fibers ( $a=0$ meaning no activation). Notice that we assumed that $\operatorname{det} \mathrm{F}_{a}=1$, so that also $\operatorname{det} \mathrm{F}_{e}=1$ and the material is elastically incompressible. Moreover, in order to accommodate the experimental data (see Fig. 1), the activation parameter $a$ cannot be constant. Indeed, it is a key feature of the skeletal muscle tissue that the active part of the stress grows with the stretch until a maximum and then decreases; this behavior is probably due to the molecular structure of the sarcomere, in which the overlap between actin and myosin depends also on the stretch. Moreover, also from the mathematical point of view it can be proved that a constant activation in the strain energy function (7) cannot reproduce such a behavior. Hence we assume that the activation parameter $a$ depends on the deformation. A reasonable choice is to take $a$ as a function of the squared stretch along the fibers, which is measured by

$$
I_{4}=\operatorname{tr}(\mathrm{CM})
$$

so that $\mathrm{F}_{a}=\mathrm{F}_{a}\left(I_{4}\right)$. In that case, taking into account that $\operatorname{det} \mathrm{F}_{a}=1$, the corresponding first Piola-Kirchhoff stress tensor is given by

$$
\widehat{\mathrm{P}}=2 \mathrm{~F} \frac{\partial \widehat{W}}{\partial \mathrm{C}}-\widehat{p} \mathrm{~F}^{-T}=2 \mathrm{~F} \frac{\partial}{\partial \mathrm{C}}\left[W\left(\mathrm{~F}_{a}^{-T}\left(I_{4}\right) \mathrm{CF}_{a}^{-1}\left(I_{4}\right)\right)\right]-\widehat{p} \mathrm{~F}^{-T}
$$

where $\widehat{p}$ accounts for the incompressibility constraint $\operatorname{det} \mathrm{C}=1$.

The experimental data of [5] are collected during a uniaxial deformation along the fibers. Hence we consider the uniform incompressible transversely isotropic deformation

$$
\mathbf{F}=\lambda \mathbf{m} \otimes \mathbf{m}+\frac{1}{\sqrt{\lambda}}(\mathbf{I}-\mathbf{m} \otimes \mathbf{m})
$$

so that $\lambda=\sqrt{I_{4}}$. Taking into account the form of the activation (8) and the expression of the energy ( 7 ), one gets

$$
\widehat{W}(\lambda, a(\lambda))=\frac{\mu}{4}\left[\frac{1}{\alpha}\left(e^{\alpha\left(I_{e}-1\right)}-1\right)+K_{e}-1\right],
$$


where

$$
\begin{gathered}
I_{e}=\frac{w_{0}}{3} \operatorname{tr}\left(\mathrm{C}_{e}\right)+\left(1-w_{0}\right) \operatorname{tr}\left(\mathrm{C}_{e} \mathrm{M}\right)=\left(1-\frac{2}{3} w_{0}\right) \frac{\lambda^{2}}{(1-a)^{2}}+\frac{2 w_{0}}{3 \lambda}(1-a), \\
K_{e}=\frac{w_{0}}{3} \operatorname{tr}\left(\mathbf{C}_{e}^{-1}\right)+\left(1-w_{0}\right) \operatorname{tr}\left(\mathbf{C}_{e}^{-1} \mathbf{M}\right)=\left(1-\frac{2}{3} w_{0}\right) \frac{(1-a)^{2}}{\lambda^{2}}+\frac{2 w_{0} \lambda}{3(1-a)}, \\
\mathbf{C}_{e}=\frac{\lambda^{2}}{(1-a)^{2}} \mathbf{m} \otimes \mathbf{m}+\frac{1-a}{\lambda}(\mathbf{I}-\mathbf{m} \otimes \mathbf{m}) .
\end{gathered}
$$

In order to accommodate the experimental data, $a(\lambda)$ should fulfill the equation

$$
P_{\text {tot }}=P_{\text {pas }}+P_{\text {act }},
$$

where $P_{\text {tot }}=\operatorname{tr}(\widehat{\mathrm{PM}})$ and $P_{\text {pas }}=\operatorname{tr}(\mathrm{PM})$ represent the component along $M$ of the first Piola-Kirchhoff stress tensor in the total (9) and passive (6) case, respectively, while $P_{a c t}$ describes the data of the active curve. In our case, we can compute $P_{\text {tot }}$ and $P_{\text {pas }}$ directly from the corresponding strain energy densities (10) and (4):

$$
\begin{gathered}
P_{\text {tot }}(\lambda, a(\lambda))=\frac{\partial \widehat{W}}{\partial \lambda}(\lambda, a(\lambda))+\frac{\partial \widehat{W}}{\partial a}(\lambda, a(\lambda)) a^{\prime}(\lambda), \\
P_{\text {pas }}(\lambda)=\frac{\partial W}{\partial \lambda}(\lambda)=\frac{\partial \widehat{W}}{\partial \lambda}(\lambda, 0) .
\end{gathered}
$$

As far as $P_{a c t}$ is concerned, it is a function of $\lambda$ which has to be deduced from the experiments. Once $P_{a c t}$ is described, the activation function $a(\lambda)$ must solve the ODE

$$
\frac{\partial \widehat{W}(\lambda, a(\lambda))}{\partial \lambda}+\frac{\partial \widehat{W}(\lambda, a(\lambda))}{\partial a} a^{\prime}(\lambda)-\frac{\partial \widehat{W}}{\partial \lambda}(\lambda, 0)=P_{a c t},
$$

with the initial condition $a\left(\lambda_{\min }\right)=0$, where $\lambda_{\min }$ is the value of the stretch at which the activation begins. We set $\lambda_{\min }=0.6$ because a fiber can shorten at about $60 \%$ of its length [15].

The previous problem is equivalent to

$$
\widehat{W}(\lambda, a(\lambda))-\widehat{W}(\lambda, 0)=S_{a c t},
$$

where $S_{a c t}$ is the primitive function of $P_{a c t}$ such that $S_{a c t}\left(\lambda_{\text {min }}\right)=0$.

In the literature, $P_{\text {act }}$ has often been modeled by a (piecewise) smooth function of $\lambda$, see $[2,9,16-18]$. This means that one has to choose constitutively a form of the function $P_{a c t}(\lambda)$ and to fit the constitutive parameters 
on the experimental data. However, since $a(\lambda)$ enters into $\widehat{W}$ in a complicated manner, it is difficult to solve equation (12) in closed form, whatever is the choice of $P_{a c t}$, so that a numerical solution is provided. Moreover, since we need a function of $\lambda$ in order to write the hyperelastic energy density $\widehat{W}$, a suitable expression of $a(\lambda)$ can be fitted on the numerical solution.

We decide to avoid the constitutive assumption on $P_{a c t}$ and to provide a model for $a(\lambda)$, so that the term $\widehat{W}(\lambda, a(\lambda))-\widehat{W}(\lambda, 0)$ is fitted directly on $S_{a c t}$, which now is a numerical integration of the data $P_{a c t}$. Then we choose $a(\lambda)$ of the form

$$
a(\lambda)=e^{-p_{6} \lambda}\left(p_{5} \lambda^{5}+p_{4} \lambda^{4}+p_{3} \lambda^{3}+p_{2} \lambda^{2}+p_{1} \lambda+p_{0}\right)\left(\lambda-\lambda_{\min }\right),
$$

where $p_{j}, j=0, \ldots, 6$ are the fitting parameters and $p_{6} \geq 0$. The presence of the exponential part ensures that the activation becomes negligible for $\lambda$ sufficiently large, as it is suggested by biological reasons. Moreover, it is clear that $a\left(\lambda_{\min }\right)=0$.

Fig. 4 shows the profile of $a(\lambda)$ obtained by least squares optimization of equation (12), where $S_{a c t}$ is computed by $P_{a c t}$ using the trapezoidal rule. The corresponding total and active stress curves are showed in Fig. 5.

With the values of the parameters given in Fig. 4, it happens that, for some values of the stretch $\lambda$, which are in any case outside the experimental range, the total stress becomes less than the passive one. Since for such values it is reasonable to assume that the passive part prevails, we truncate the function $a(\lambda)$.

\section{Modeling sarcopenia}

We now want to describe from a mathematical point of view a skeletal muscle tissue damaged for the presence of sarcopenia. This syndrome is defined as the age-related decrease of muscle mass and force [19]. We recall that muscle strength does not depend only on muscle mass and the relationship between strength and mass is not linear. Hence, our model should include two different types of damage: one for the loss of activation and one for the loss of mass.

\subsection{Loss of activation}

In $[20,21]$ it is remarked that aging is associated with changes in muscle mass, composition, activation and material properties. In sarcopenic muscle, there is a loss of motor units via denervation and a net conversion from fast to slow fibers, with a resulting loss in muscle power. Hence, the loss of performance of a sarcopenic muscle can be described as a weakening of the activation of the fibers. However, one of the main problem in detecting 


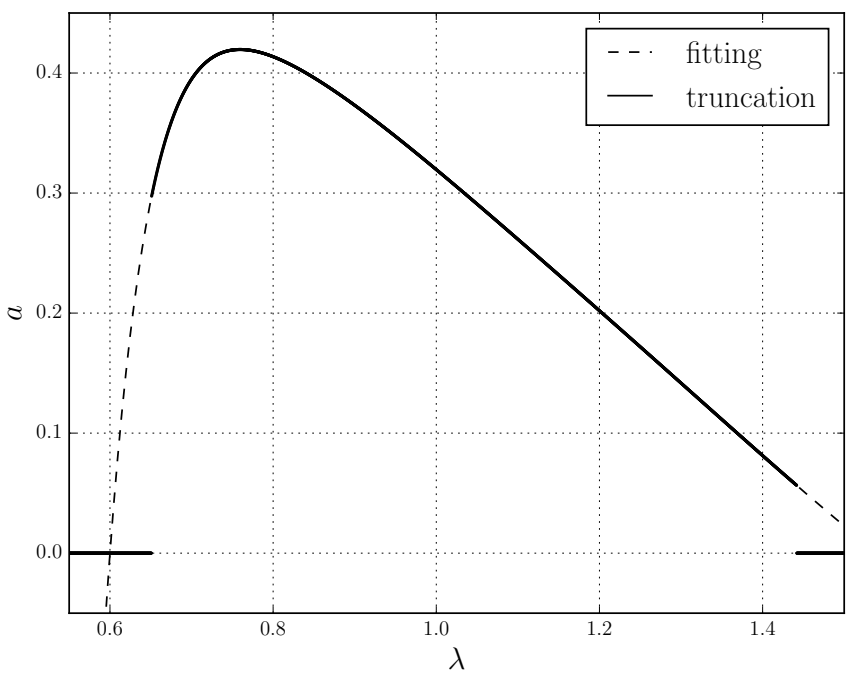

Figure 4. The activation function (13) with $p_{6}=5.408, p_{5}=-4766, p_{4}=23592$, $p_{3}=-47518, p_{2}=48938, p_{1}=-25658, p_{0}=5590$. The truncation prevents the total stress to become less than the passive one.

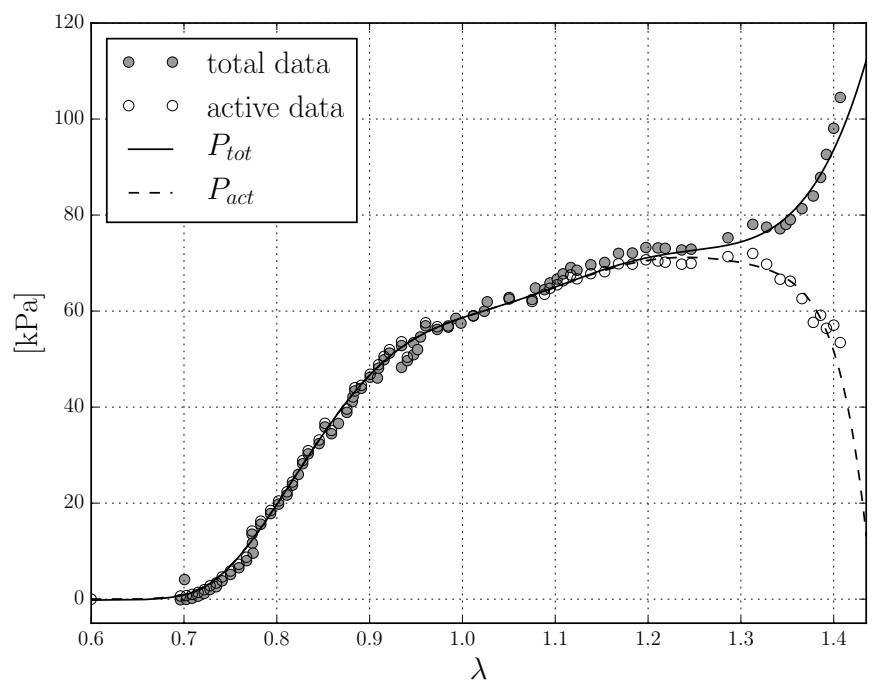

Figure 5. Total and active stress-strain relations. 
and diagnosing sarcopenia is the lack of experimental data, so that only some simple strategies can be reasonably adopted to describe the loss of performance of a sarcopenic muscle.

Following [2], we include in the model a parameter $d$ which weakens the active part of the stress along the fibers, so that the active stress of the damaged muscle becomes

$$
(1-d) P_{a c t}
$$

The dimensionless parameter $d \in[0,1]$ describes the percentage of disease or damage in the activation ability of the muscle: if $d=0$, then the muscle is healthy and can be fully activated.

We now would like to see what is the effect of a constant damage $d$ on the response of the whole muscle. In our case, lowering the active stress data $P_{\text {act }}$ means that we have to lower its integral $S_{\text {act }}$ by the factor $1-d$. Hence, instead of solving (12), at a certain $d$ we have to solve

$$
\widehat{W}(\lambda, a(\lambda))-\widehat{W}(\lambda, 0)=(1-d) S_{a c t} .
$$

The numerical solution $a(\lambda)$ is again assumed of the form (13) and it depends on the value of the parameter $d$. When $d$ increases, Fig. 6 shows the behavior of $a(\lambda)$ obtained by the least squares optimization and the corresponding stress-stretch relations are plotted in Fig. 7.

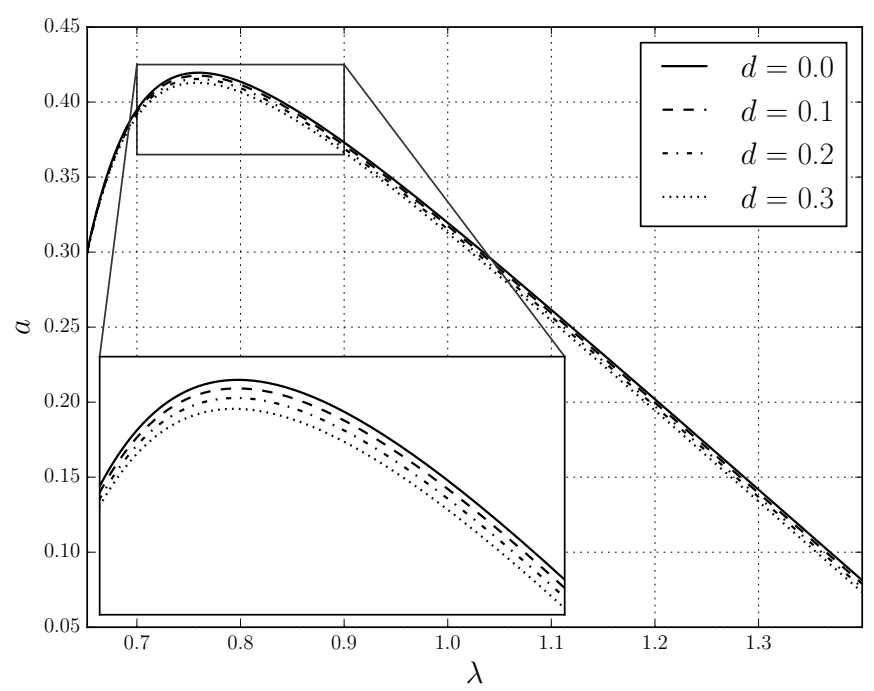

Figure 6. Effects of the loss of activation on $a(\lambda)$. 


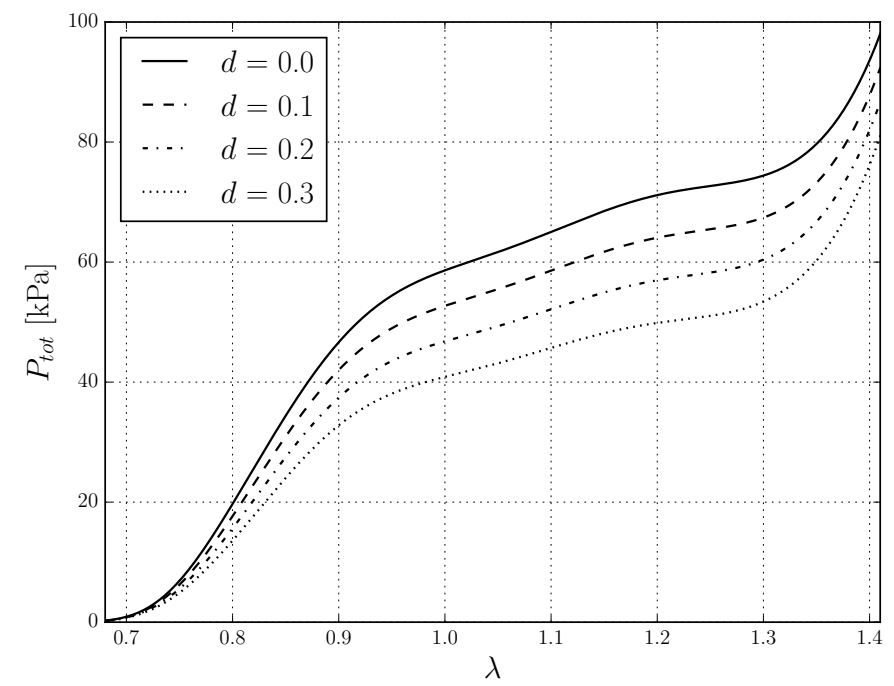

Figure 7. Effects of the loss of activation on the total stress. Fitting parameters are not reported.

As one would expect, when $d$ increases the activation $a(\lambda)$ decreases. It means that lowering the curve of $P_{\text {act }}$ results in a decrease of $a(\lambda)$, which leads to a lowered total stress response (Fig. 7).

The damage parameter mainly affects the value of the active stress in the region near its maximum and the qualitative behavior of the total stress curve does not change, at least for $d \leq 0.5$. Beyond the region where the active part prevails, the stress follows the exponential growth of the passive curve.

\subsection{Loss of mass}

Let us consider the other main feature of sarcopenia, namely the loss of mass. This issue can be addressed by following growth theory and plasticity [22].

Similar to the active strain approach explained in Section 2.2, we rewrite the deformation gradient as

$$
\mathrm{F}=\mathrm{F}_{e} \mathrm{~F}_{a} \mathrm{~F}_{g}
$$

where $\mathrm{F}_{e}$ is the elastic part, $\mathrm{F}_{a}\left(I_{4}\right)$ describes the active contribution as before, while $\mathrm{F}_{g}$ represents the loss of mass (Fig. 8).

The volume elements are modified by the internal active forces and by the loss of mass without changing the elastic energy, so that the strain 


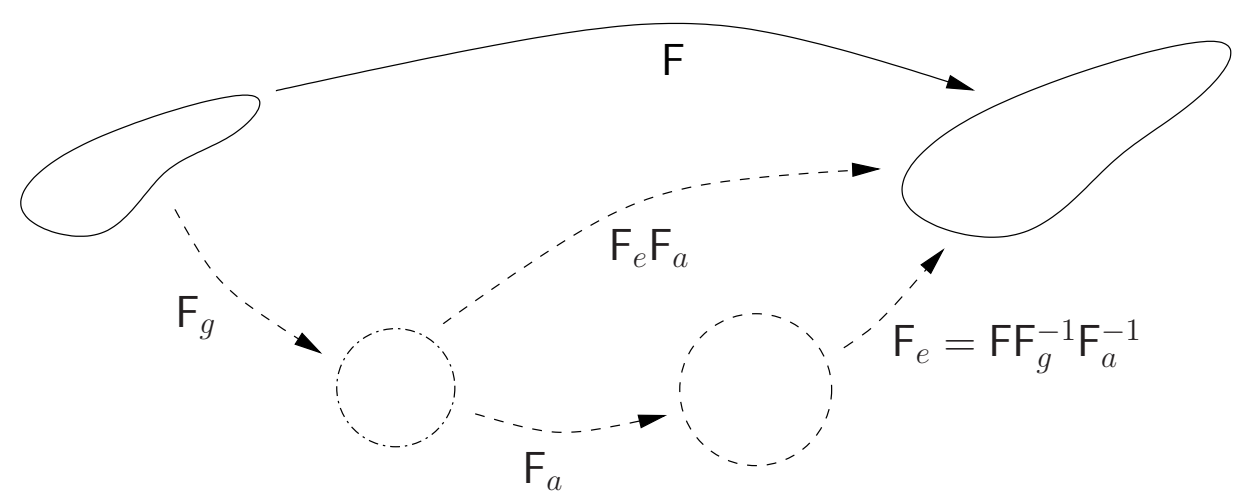

Figure 8. A pictorial description of the loss of mass.

energy function of the material has to be computed using $\mathrm{C}_{e}=\mathrm{F}_{e}^{T} \mathrm{~F}_{e}$, where $\mathrm{F}_{e}=\mathrm{FF}_{g}^{-1} \mathrm{~F}_{a}^{-1}$. We then obtain the modified hyperelastic energy density

$$
\widetilde{W}(\mathrm{C})=\left(\operatorname{det} \mathrm{F}_{g}\right) \widehat{W}\left(\mathrm{~F}_{g}^{-T} \mathrm{CF}_{g}^{-1}\right)=\left(\operatorname{det} \mathrm{F}_{a} \mathrm{~F}_{g}\right) W\left(\mathrm{~F}_{a}^{-T} \mathrm{~F}_{g}^{-T} \mathrm{CF}_{g}^{-1} \mathrm{~F}_{a}^{-1}\right) .
$$

Moreover, we underline that now $\mathrm{F}$ is compressible due to the loss of mass described by $\mathrm{F}_{g}$; in this case, the map $\mathrm{F}_{e} \mathrm{~F}_{a}$ has to be incompressible, which, thanks to the diagram in Fig. 8, provides the incompressibility of the map $\mathrm{FF}_{g}^{-1}$, that is $\operatorname{det} \mathrm{F}=\operatorname{det} \mathrm{F}_{g}$. In terms of $\mathrm{C}$ the constraint becomes

$$
\operatorname{det} \mathrm{F}_{g}^{-2} \operatorname{det} \mathrm{C}=1 \text {. }
$$

Taking into account all the previous arguments, the corresponding first Piola-Kirchhoff stress tensor is given by

$$
\begin{aligned}
\widetilde{\mathrm{P}} & =2 \mathrm{~F} \frac{\partial \widetilde{W}}{\partial \mathrm{C}}-\widetilde{p} \frac{\partial}{\partial \mathrm{C}}\left(\operatorname{det} \mathrm{F}_{g}^{-2} \operatorname{det} \mathrm{C}-1\right) \\
& =2\left(\operatorname{det} \mathrm{F}_{g}\right) \mathrm{F} \frac{\partial}{\partial \mathrm{C}}\left[\operatorname{det} \mathrm{F}_{a}\left(I_{4}\right) W\left(\mathrm{~F}_{a}^{-T}\left(I_{4}\right) \mathrm{F}_{g}^{-T} \mathrm{CF}_{g}^{-1} \mathrm{~F}_{a}^{-1}\left(I_{4}\right)\right)\right]-\widetilde{p}\left(\operatorname{det} \mathrm{F}_{g}^{-2}\right) \mathrm{F}^{-T},
\end{aligned}
$$

where $\widetilde{p}$ accounts for the incompressibility constraint (16).

The active contribution $\mathrm{F}_{a}\left(I_{4}\right)$ is modeled in Section 2.2, while we have to specify the form of $F_{g}$. Since by aging the number of fibers decreases, we describe a loss of mass in the direction orthogonal to the fibers by choosing

$$
\mathrm{F}_{g}=\mathbf{m} \otimes \mathbf{m}+\sqrt{1-g}(\mathrm{I}-\mathbf{m} \otimes \mathbf{m}),
$$

where $0 \leq g<1$ is a dimensionless parameter representing the percentage of loss ( $g=0$ meaning no loss of mass). 
It is interesting to look at the stress-strain curve for a uniaxial deformation along the fibers when the parameter $g$ varies. As in the previous sections, let us consider a transversely isotropic deformation along the fibers; since the global deformation now is no more incompressible and $\operatorname{det} \mathrm{F}=\operatorname{det} \mathrm{F}_{g}$, then the deformation gradient writes

$$
\mathbf{F}=\lambda \mathbf{m} \otimes \mathbf{m}+\sqrt{\frac{1-g}{\lambda}}(\mathbf{I}-\mathbf{m} \otimes \mathbf{m}),
$$

so that

$$
\mathrm{C}_{e}=\frac{\lambda^{2}}{(1-a)^{2}} \mathbf{m} \otimes \mathbf{m}+\frac{1-a}{\lambda}(\mathrm{I}-\mathbf{m} \otimes \mathbf{m}),
$$

as in Section 2.2. Hence, from (10) and (15), the corresponding strain energy function is

$$
\widetilde{W}(\lambda, a(\lambda), g)=(1-g) \widehat{W}(\lambda, a(\lambda)) .
$$

From the previous identity it is clear that, whenever $g>0$, we are lowering the behavior of the total stress by a factor $1-g$, as it is showed in Fig. 9.

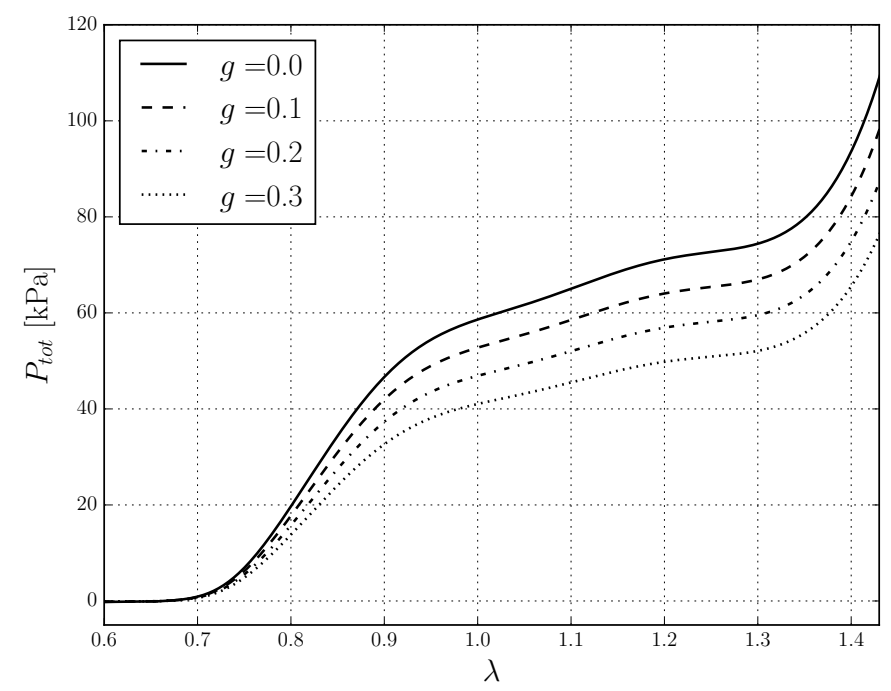

Figure 9. Effects of the loss of mass on the total stress. 


\subsection{A comparison: $g=d$}

Figures 7 and 9 reveal that both the parameters $d$ and $g$ decrease the behavior of the total stress, as one would expect in sarcopenia. Actually, the lowering effects in the two cases are very similar and it is interesting to compare them.

In Section 3.1, the damage of the activation state of the fibers is included in the model by weakening the active curve with the term $1-d$. On the other hand, the loss of mass appears in the energy (18) only by the multiplicative factor $1-g$. Fig. 10 shows the behavior of the stress-strain curve when the parameters $d$ and $g$ decrease. The plots of the two kinds of damage overlap almost perfectly when $P_{a c t}$ is large, while the two curves branch out in the region where $P_{\text {pas }}$ prevails over $P_{\text {act }}$. The reason of this behavior relies on the way the two damages are included in the model: $g$ affects all the hyperelastic energy in (18), while $d$ lowers only the active part of the stress (14).

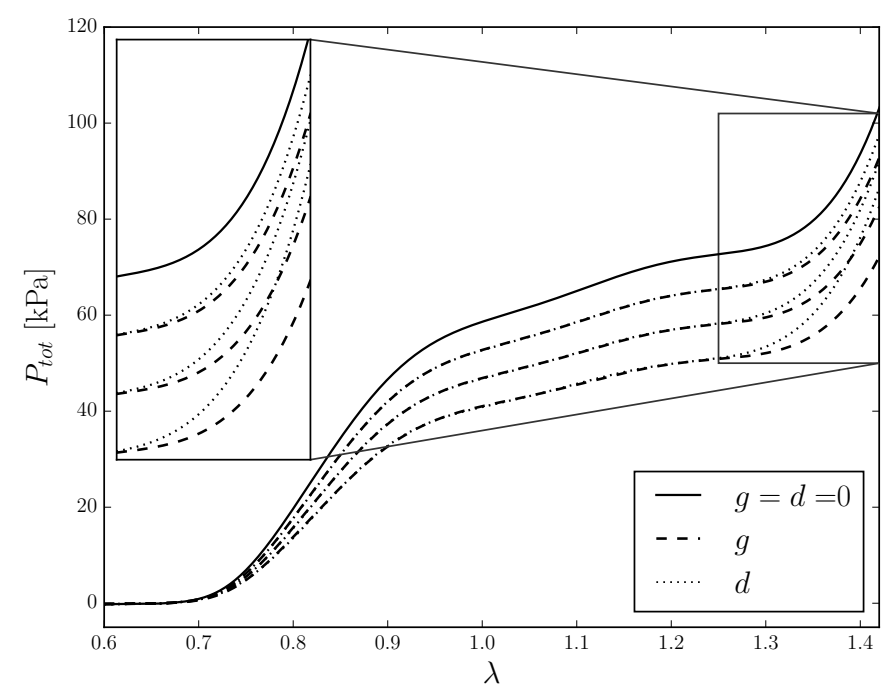

Figure 10. A comparison between loss of activation and loss of mass when $g=d$.

\section{Conclusions}

We introduced a hyperelastic model for skeletal muscle tissue which can keep into account the loss of mass and the loss of activation, two typical symptoms of the syndrome named sarcopenia.

The passive strain energy density of the material is of exponential type 
and keeps into account the preferred direction of the muscle fibers, modeling the material as transversely isotropic, while the incompressibility, which is due to the high water content, has been introduced as an external constraint. The activation of the material has been modeled by the active strain approach, and an activation function of the stretch along the muscle fibers has been deduced. Such an activation, being the solution of a transcendental equation, is not written in closed form, but a suitable function is fitted on the numerical data. The model has a very good agreement with the experiments on a rat tibialis anterior. The dependence of the activation on the stretch along the fibers is one of the novelties of our approach; it allows to describe the active muscle tissue as a true hyperelastic material.

The other main feature of our model is to consider the presence of the sarcopenia by means of two parameters: a percentage of damage $d$ which lowers the active part of the stress and a percentage of loss of mass $g$ which is included by a multiplicative decomposition of the deformation gradient. Moreover, the effects of the two parameters have been discussed and compared. Namely, both the parameters induce a reduction of the stress response of the tissue, see Figs. 7 and 9, as it happens in a sarcopenic muscle, although the effect of the loss of mass is much more evident at large values of the stretch.

The model is developed fully $3 \mathrm{D}$ and the proposed hyperelastic energy is appropriate for a finite element implementation, hence it is easy to produce numerical simulations which go beyond the uniaxial case, such as simple shear or biaxial loading. However, the lack of experimental data for such deformations does not allow to compare the results with any real case.

An ongoing work is the application of the model to a realistic threedimensional muscle geometry. Another goal is to relate the parameters $d, g$ with some chemical or physiological factors, and to give a quantitative description of their evolution during aging; this is a major issue, since the molecular aspects of sarcopenia are still not fully understood. Moreover, in view of a dynamical description of muscles, a further improvement will be to take into account also the viscoelastic properties of the tissue.

\section{Acknowledgements}

This work has been supported by the project Active Ageing and Healthy Living of the Università Cattolica del Sacro Cuore and partially supported by National Group of Mathematical Physics (GNFM-INdAM). 


\section{REFERENCES}

1. A. Musesti, G. G. Giusteri, and A. Marzocchi, Predicting Ageing: On the Mathematical Modelization of Ageing Muscle Tissue, in Active Ageing and Healthy Living (G. R. et al., ed.), IOS press, 2014. Chapter 17.

2. G. Giantesio and A. Musesti, A continuum model of skeletal muscle tissue with loss of activation, in Multiscale Models in Mechano and Tumor Biology: Modeling, Homogenization, and Applications (A. Gerisch, R. Penta, and J. Lang, eds.), vol. 122 of Lecture Notes in Computational Science and Engineering, Springer, in press.

3. L. A. Taber and R. Perucchio, Modeling heart development, Journal of Elasticity, vol. 61, no. 1, pp. 165-197, 2000.

4. G. Giantesio and A. Musesti, Strain-dependent internal parameters in hyperelastic biological materials, International Journal of Non-Linear Mechanics, vol. 95, pp. 162-167, 2017.

5. D. Hawkins and M. Bey, A Comprehensive Approach for Studying Muscle-Tendon Mechanics, ASME Journal of Biomechanical Engineering, vol. 116, pp. 51-55, 1994.

6. R. L. Lieber and S. R. Ward, Skeletal muscle design to meet functional demands, Philosophical Transactions of the Royal Society of London B: Biological Sciences, vol. 366, no. 1570, pp. 1466-1476, 2011.

7. J. Schröder and P. Neff, Invariant formulation of hyperelastic transverse isotropy based on polyconvex free energy functions, International Journal of Solids and Structures, vol. 40, pp. 401-445, 2003.

8. A. E. Ehret and M. Itskov, Modeling of anisotropic softening phenomena: Application to soft biological tissues, International Journal of Plasticity, vol. 25, pp. 901-919, 2009.

9. A. E. Ehret, M. Böl, and M. Itskov, A continuum constitutive model for the active behaviour of skeletal muscle, Journal of the Mechanics and Physics of Solids, vol. 59, no. 3, pp. 625-636, 2011.

10. A. E. Ehret and M. Itskov, A polyconvex hyperelastic model for fiberreinforced materials in application to soft tissues, Journal of Materials Science, vol. 42, pp. 8853-8863, 2007.

11. P. Nardinocchi and L. Teresi, On the Active Response of Soft Living Tissues, Journal of Elasticity, vol. 88, no. 1, pp. 27-39, 2007.

12. D. Ambrosi and S. Pezzuto, Active Stress vs. Active Strain in Mechanobiology: Constitutive Issues, Journal of Elasticity, vol. 107, pp. 199-212, 2012.

13. A. Gizzi, C. Cherubini, S. Filippi, and A. Pandolfi, Theoretical and numerical modeling of nonlinear electromechanics with applications to biological active media, Communications in Computational Physics, vol. 17 , no. 1, pp. 93-126, 2015. 
14. A. Gizzi, A. Pandolfi, and M. Vasta, Viscoelectromechanics modeling of intestine wall hyperelasticity, International Journal for Computational Methods in Engineering Science and Mechanics, vol. 17, no. 3, pp. 143$155,2016$.

15. C. A. Oatis, Kinesiology. The Mechanics and Pathomechanics of Human Movement. Lippincott Williams \& Wilkins, 2nd ed., 2009.

16. J. L. van Leeuwen, Optimum power output and structural design of sarcomeres, Journal of Theoretical Biology, vol. 149, pp. 229-256, 1991.

17. M. Böl and S. Reese, Micromechanical modelling of skeletal muscles based on the finite element method, Computer Methods in Biomechanics and Biomedical Engineering, vol. 11, pp. 489-504, 2008.

18. T. Heidlauf and O. Röhrle, A multiscale chemo-electro-mechanical skeletal muscle model to analyze muscle contraction and force generation for different muscle fiber arrangements, Frontiers in Physiology, vol. 5, p. 498, 2014.

19. A. J. Cruz-Jentoft, J. P. Baeyens, J. M. Bauer, Y. Boirie, T. Cederholm, F. Landi, F. C. Martin, J. P. Michel, Y. Rolland, S. M. Schneider, E. Topinková, M. Vandewoude, and M. Zamboni, Sarcopenia: European consensus on definition and diagnosis, Age and Ageing, vol. 39, pp. 412$423,2010$.

20. T. Lang, T. Streeper, P. Cawthon, K. Baldwin, D. R. Taaffe, and T. B. Harris, Sarcopenia: etiology, clinical consequences, intervention, and assessment, Osteoporosis International, vol. 21, pp. 543-559, 2010.

21. S. von Haehling, J. E. Morley, and S. D. Anker, An overview of sarcopenia: facts and numbers on prevalence and clinical impact, Journal of Cachexia, Sarcopenia and Muscle, vol. 1, pp. 129-133, 2010.

22. A. DiCarlo and S. Quiligotti, Growth and balance, Mechanics Research Communications, vol. 29, no. 6, pp. 449-456, 2002. 